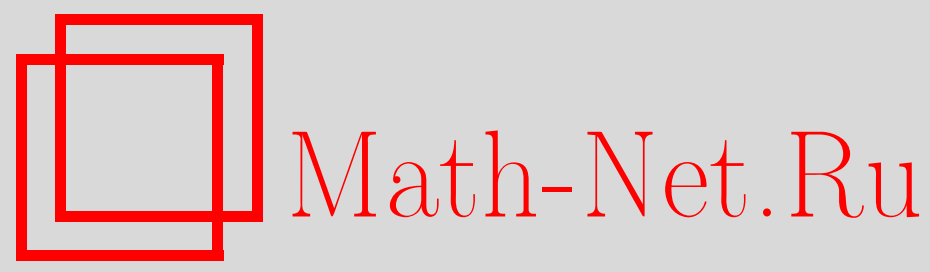

C. С. Марченков, Позитивно замкнутые классы трехзначной логики, порождаемые одноместными функциями, Дискрет. матем., 2009, том 21, выпуск 3, 37-44

DOI: https://doi.org/10.4213/dm1060

Использование Общероссийского математического портала Math-Net.Ru подразумевает, что вы прочитали и согласны с пользовательским соглашением http://www. mathnet.ru/rus/agreement

Параметры загрузки:

IP : 3.82 .47 .9

26 апреля 2023 г., $15: 42: 23$ 
УДК 519.7

\title{
Позитивно замкнутые классы трехзначной логики, порождаемые одноместными функциями
}

\author{
() 2009 г. . С. С. Марченков
}

\begin{abstract}
На множестве $P_{3}$ функций трехзначной логики рассматривается оператор позитивного замыкания. Устанавливается, что в $P_{3}$ имеется ровно 51 позитивно замкнутый класс, позитивно порождаемый одноместными функциями. Из них 26 классов порождаются единственной одноместной функцией (включая один из позитивно предполных в $P_{3}$ классов), а 25 классов - двумя функциями (включая класс $P_{3}$ и остальные 9 позитивно предполных классов).

Работа выполнена при поддержке Российского фонда фундаментальных исследований, проект 06-01-00438.
\end{abstract}

\section{1. Введение}

Один из возможных способов классификации множества $P_{k}$ функций $k$-значной логики состоит в задании на множестве $P_{k}$ оператора замыкания $\mathrm{Cl}$. Совокупность всех классов (как правило, пересекающихся), замкнутых относительно оператора $\mathrm{Cl}$, образует Cl-классификацию множества $P_{k}$. Известно, что для оператора суперпозиции соответствующая классификация множества $P_{2}$ счетна $[7,8]$, а при $k \geqslant 3$ классификация множества $P_{k}$ континуальна [6]. В связи с этим представляют интерес “сильные” операторы замыкания, которые при любом $k \geqslant 3$ приводят к конечным либо счетным классификациям.

В работе [2] автором введен оператор Роs позитивного замыкания и установлено, что при любом $k \geqslant 2$ число позитивно замкнутых классов в $P_{k}$ конечно. Исследование оператора позитивного замыкания было продолжено в работах $[3,4]$. В частности, в $P_{3}$ были найдены все (их оказалось 10) позитивно предполные классы [3], а также все (в количестве 17) дискриминаторные позитивно замкнутые классы [4]. При этом обнаружилось, что в $P_{3}$ многие довольно крупные позитивно замкнутые классы позитивно порождаются множествами одноместных функций. Это справедливо, например, для класса $P_{3}$ и всех предполных в нем классов [3]. Однако, предположение о том, что все позитивно замкнутые классы в $P_{3}$ имеют позитивные базисы, состоящие из одноместных функций, неверно - опровержением может служить часть из дискриминаторных классов [4].

Ряд фактов, найденных при исследовании позитивно замкнутых классов в $P_{3}$, указывает на то, что, по-видимому, большинство позитивно замкнутых классов в $P_{3}$ все же позитивно порождаются множествами одноместных функций. В настоящей работе мы описываем все 51 позитивно замкнутых класса в $P_{3}$, которые имеют позитивные базисы из одноместных функций. Большая часть из этих классов описывается впервые. При 
доказательстве отделимости найденных замкнутых классов используются два приема: отделение с помощью пересечений позитивно предполных классов и отделение с помощью полугрупп эндоморфизмов.

\section{2. Основные понятия}

Пусть $E_{3}=\{0,1,2\}, P_{3}-$ множество всех функций на $E_{3}$ (множество функций трехзначной логики). Если $Q \subseteq P_{3}$, то через $Q^{(1)}$ обозначаем множество всех одноместных функций из $Q$. Для функций $g \in P_{3}^{(1)}$ в дальнейшем используем обозначение $(g(0) g(1) g(2))$. Например, (210) есть функция из $P_{3}^{(1)}$, принимающая значения $2,1,0$ соответственно при $x=0,1,2$.

Пусть $\pi-$ перестановка на $E_{3}$ и $g \in P_{3}$. Функция

$$
g^{\pi}\left(x_{1}, \ldots, x_{n}\right)=\pi^{-1}\left(g\left(\pi\left(x_{1}\right), \ldots, \pi\left(x_{n}\right)\right)\right)
$$

называется двойственной к функции $g$ относительно перестановки $\pi$. Если $g=g^{\pi}$, то функция $g$ называется самодвойственной относительно перестановки $\pi$. Множества всех функций из $P_{3}$, самодвойственных относительно перестановок $x+1,2 \mathrm{x}, 2 x+2,2 x+1$ (здесь и далее сложение и умножение рассматриваются по модулю 3 ), обозначим через $S_{x+1}, S_{2 x}, S_{2 x+2}, S_{2 x+1}$ соответственно.

Пусть $T_{0}, T_{1}, T_{2}$ обозначают множества всех функций из $P_{3}$, сохраняющих соответственно $0,1,2$. Положим

$$
T_{01}=T_{0} \cap T_{1}, \quad T_{02}=T_{0} \cap T_{2}, \quad T_{12}=T_{1} \cap T_{2} .
$$

Напомним основные понятия, связанные с оператором позитивного замыкания [2]. Вначале определим язык Pos. Исходными символами языка Pos являются символы предметных переменных $x_{1}, x_{2}, \ldots$ (с областью значений $\left.E_{3}\right)$, символы $f_{i}^{(n)}$ для обозначения $n$-местных функций из $P_{3}$, знаки равенства $=$, конъюнкции $\&$, дизъюнкции $\vee$, квантор существования $\exists$, левая и правая скобки и запятая. Иногда вместо символов переменных $x_{1}, x_{2}, \ldots$ будем использовать символы $x, y, z$.

Терм в языке Pos определим по индукции. Символ предметной переменной есть терм; если $x_{j_{1}}, \ldots, x_{j_{n}}-$ символы предметных переменных (не обязательно различные), а $f_{i}^{(n)}$ - символ $n$-местной функции, то $f_{i}^{(n)}\left(x_{j_{1}}, \ldots, x_{j_{n}}\right)$ есть терм; если $t_{1}, \ldots, t_{m}$ - термы, а $f_{l}^{(m)}$ - символ $m$-местной функции, то $f_{l}^{(m)}\left(t_{1}, \ldots, t_{m}\right)$ есть терм. Других термов в языке Pos нет.

Всякий терм языка Pos очевидным образом определяет некоторую функцию класса $P_{3}$ (переменная определяет тождественную функцию).

Если $t_{1}, t_{2}$ - термы языка Pos, то выражение $\left(t_{1}=t_{2}\right)$ называем элементарной формулой языка Pos. Далее, если $\Phi_{1}, \Phi_{2}-$ формулы языка Pos, а $x_{i}$ - символ предметной переменной, то $\left(\Phi_{1} \& \Phi_{2}\right),\left(\Phi_{1} \vee \Phi_{2}\right),\left(\exists x_{i}\right) \Phi_{1}-$ также формулы языка Роs. Понятия свободной и связанной переменных предполагаем известными.

Всякая формула языка Pos c $m$ свободными переменными определяет некоторое $m$ местное отношение на $E_{3}$. Пусть $Q \subseteq P_{3}, \Phi\left(x_{1}, \ldots, x_{m}\right)$ - формула языка Роs со свободными переменными $x_{1}, \ldots, x_{m}$, все функциональные символы которой суть обозначения функций из $Q$, и формула $\Phi\left(x_{1}, \ldots, x_{m}\right)$ определяет отношение $\rho\left(x_{1}, \ldots, x_{m}\right)$ на $E_{3}$. В этом случае говорим, что формула $\Phi$ позитивно выражает отношение $\rho$ через функции множества $Q$. Понятие позитивной выразимости перенесем с отношений на функции. 
Именно, если $g\left(x_{1}, \ldots, x_{m}\right)$ - функция из $P_{3}$, а формула $\Phi\left(x_{1}, \ldots, x_{m}, y\right)$ языка Pos позитивно выражает отношение $g\left(x_{1}, \ldots, x_{m}\right)=y$ (график функции $g$ ) через функции множества $Q$, то говорим, что формула $\Phi$ позитивно выражает функцию $g$ через функции множества $Q$. Совокупность всех функций, позитивно выразимых через функции множества $Q$, называем позитивным замыканием множества $Q$ и обозначаем $\operatorname{Pos}[Q]$. Множества вида $\operatorname{Pos}[Q]$ называем позитивно замкнутыми классами.

Известные для операции суперпозиции понятия полноты, порождающей системы и базиса [5] распространяем на оператор позитивного замыкания. Отметим [2], что любой позитивно замкнутый класс содержит тождественную функцию $x$ и замкнут относительно операции суперпозиции. Кроме того, в [3] доказано, что для любой перестановки $\pi$ и любого $i$ классы $T_{i}$ множества $S_{\pi}$ позитивно замкнуты. Позитивно замкнутым будет и пересечение любого числа таких классов.

Определим классы $V_{01}, V_{02}, V_{10}, V_{12}, V_{20}, V_{21}$ как позитивные замыкания следующих множеств одноместных функций: $\{0,(002),(010),(220), 2\},\{0,(002),(010),(101), 1\}$, $\{(011), 1,(112),(221), 2\},\{0,(011),(100), 1,(112)\},\{(022), 1,(121),(212), 2\},\{0,(022)$, (200), (212), 2\}. В [3] показано, что классы $V_{01}, V_{02}, V_{10}, V_{12}, V_{20}, V_{21}$ являются позитивно предполными в $P_{3}$, а множества их одноместных функций состоят из функций представленных выше порождающих систем и тождественной функции $x$.

\section{3. Предварительные результаты}

В дальнейшем мы неоднократно пользуемся следующим принципом двойственности для позитивной выразимости, который установлен в работе [2] (его формулировку приводим для класса $\left.P_{3}\right)$.

Пусть $\pi$ - перестановка на множестве $E_{3},\left\{g_{1}, \ldots, g_{m}\right\} \subset P_{3}$ и формула $\Phi$ языка Роs позитивно выражает функцию $g$ через функции $g_{1}, \ldots, g_{m}$. Тогда функция $g^{\pi}$ позитивно выразима через функции $g_{1}^{\pi}, \ldots, g_{m}^{\pi}$ формулой $\Phi^{\pi}$, которая получается из формулы $\Phi$ заменой всех символов функций $g_{1}, \ldots, g_{m}$ соответственно символами двойственных функций $g_{1}^{\pi}, \ldots, g_{m}^{\pi}$.

Пусть $Q \subseteq P_{3}^{(1)}$. Если $Q$ целиком не содержится ни в одном из десяти позитивно предполных в $P_{3}$ классов $S_{x+1}, T_{0}, T_{1}, T_{2}, V_{01}, V_{02}, V_{10}, V_{12}, V_{20}, V_{21}$, то, согласно критерию позитивной полноты [3], множество $Q$ является позитивно полным в $P_{3}$. Поэтому далее будем рассматривать лишь такие множества $Q$, которые целиком входят хотя бы в один из позитивно предполных классов.

Пусть $Q \subseteq S_{x+1}^{(1)}$. Поскольку $S_{x+1}^{(1)}=\{x, x+1, x+2\}$, множество $Q$ либо включает одну из функций $x+1, x+2$ (на самом деле одновременно обе), либо состоит только из функции $x$. Однако каждая из функций $x+1, x+2$ позитивно порождает класс $S_{x+1}$ (см. [3]). Таким образом, либо $\operatorname{Pos}[Q]=S_{x+1}$, либо $\operatorname{Pos}[Q]=\operatorname{Pos}[x]$. Отметим, что $\operatorname{Pos}[x]$ есть наименьший позитивно замкнутый класс в $P_{3}$.

При рассмотрении случаев $Q \subseteq T_{i}^{(1)}, i=0,1,2$, мы бы хотели сразу исключить из множеств $Q$ функции $2 x, 2 x+2,2 x+1$. Это можно сделать на основе следующих соображений. Функции $2 x, 2 x+2,2 x+1$ образуют позитивные базисы соответственно классов $S_{2 x}, S_{2 x+2}, S_{2 x+1}$ (см. [4]). Эти классы непосредственно содержатся соответственно только в позитивно предполных классах $T_{0}, T_{1}, T_{2}$ (см. [4]). Значит, множество $Q$, содержащее, например, функцию $2 x$, позитивно порождает либо класс $S_{2 x}$, либо класс $T_{0}$, либо класс $P_{3}$.

Итак, в дальнейшем будем предполагать, что ни одна из функций $2 x, 2 x+2,2 x+1$ 
не входит в множество $Q$.

Из трех двойственных друг другу классов $T_{0}, T_{1}, T_{2}$ рассмотрим только класс $T_{0}$. Выпишем все функции множества $T_{0}^{(1)}$, отличные от функций $x, 2 x$ :

$$
\text { 0, (001), (002), (010), (011), (020), (022). }
$$

Лемма 1. Каждая из систем функций

$$
\begin{array}{llll}
\{(001),(002)\}, & \{(001),(010)\}, & \{(001),(011)\}, & \{(001),(020)\}, \\
\{(001),(022)\}, & \{(002),(011)\}, & \{(002),(020)\}, & \{(010),(020)\}, \\
\{(010),(022)\}, & \{(011),(020)\}, & \{(011),(022)\}, & \{(020),(022)\}
\end{array}
$$

позитивно порождает класс $T_{0}$.

Доказательство. Начнем с системы $\{(001),(002)\}$. Суперпозицией функции (001) получаем константу 0. Далее,

$$
(y=2 x) \equiv(\exists z)(((001)(z)=x) \&((002)(z)=y)) \vee((002)(x)=x) \&(y=(001)(x)) .
$$

Таким образом, $S_{2 x} \subset \operatorname{Pos}[(001),(002)]$. Как отмечалось выше, класс $S_{2 x}$ непосредственно содержится только в позитивно предполном классе $T_{0}$. Поскольку $(001) \notin S_{2 x}$, получаем, что $\operatorname{Pos}[(001),(002)]=T_{0}$.

В случае системы $\{(001),(010)\}$ получаем функцию (002):

$$
(y=(002)(x)) \equiv((001)(x)=0) \&(y=0) \vee((010)(x)=0) \&(y=x) .
$$

В случае системы $\{(001),(011)\}$ получаем функцию (010):

$$
(y=(010)(x)) \equiv((001)(x)=0) \&(y=x) \vee((001)(x)=(011)(x)) \&(y=0) .
$$

В случае системы $\{(001),(020)\}$ получаем функцию (002):

$$
(y=(002)(x)) \equiv((001)(x)=0) \&(y=0) \vee((020)(x)=0) \&(y=x) .
$$

В случае системы $\{(001),(022)\}$ получаем функцию (020):

$$
(y=(020)(x)) \equiv((001)(x)=0) \&(y=(022)(x)) \vee((022)(x)=x) \&(y=0) .
$$

В случае системы $\{(002),(011)\}$ получаем функцию (001):

$$
(y=(001)(x)) \equiv((002)(x)=0) \&(y=0) \vee((002)(x)=x) \&(y=(011)(x)) .
$$

Остальные системы списка (2) двойственны рассмотренным системам относительно перестановки $2 x$ (класс $T_{0}$ перестановкой $2 x$ переводится в себя). Лемма доказана.

Список (2) содержит все пары ненулевых функций из (1) за исключением трех пар $\{(002),(010)\},\{(002),(022)\},\{(010),(011)\}$. Однако, как установлено в [4], система $\{(002),(022)\}$ позитивно порождает класс $T_{02}$, а система $\{(010),(011)\}-$ класс $T_{01}$. Кроме того, класс $T_{02}$ непосредственно содержится только в позитивно предполных классах $T_{0}$ и $T_{2}$, а класс $T_{01}-$ в классах $T_{0}$ и $T_{1}$. Следовательно, дальнейшие расширения систем $\{(002),(022)\}$ и $\{(010),(011)\}$ функциями из (1) приводят к позитивно полным множествам в классе $T_{0}$. 
Рассмотрим систему $\{(002),(010)\}$. Очевидно, что константа 0 получается суперпозицией функций (002) и (010). Заметим далее, что 0, (002), (010) суть все функции из множества $T_{0}^{(1)} \cap V_{01}^{(1)}$, отличные от $x$. Следовательно, множество всех одноместных функций позитивного замыкания системы $\{(002),(010)\}$ будет состоять в точности из функций 0, (002), (010), x. Дальнейшие расширения этого множества функциями из (1) в силу леммы 1 дают множества, позитивно полные в классе $T_{0}$.

Обратимся теперь к системам $\{0,(002)\},\{0,(010)\},\{0,(011)\},\{0,(022)\}$. Системы $\{0,(002)\},\{0,(010)\}$ позитивно порождают тот же класс, что и система $\{(002),(010)\}$, поскольку

$$
(y=(010)(x)) \equiv((002)(x)=0) \&(y=x) \vee((002)(x)=x) \&(y=0)
$$

и система $\{0,(010)\}$ двойственна системе $\{0,(002)\}$ относительно перестановки $2 x$.

Функции $0,(011)$ вместе с функцией $x$ образуют множество $T_{0}^{(1)} \cap V_{12}^{(1)}$. Поэтому множество всех одноместных функций из $\operatorname{Pos}[0,(011)]$ будет совпадать с множеством $T_{0}^{(1)} \cap V_{12}^{(1)}$. Данное свойство является характеристическим для позитивного замыкания $\operatorname{Pos}[0,(011)]$. Дальнейшие расширения системы $\{0,(011)\}$ функциями из списка (1) исследованы выше.

Система $\{0,(022)\}$ двойственна системе $\{0,(011)\}$ относительно перестановки $2 x$.

Оставшиеся две системы $\{0,(001)\},\{0,(020)\}$ из двух функций будут исследованы в разделе 3. Отметим лишь, что с точки зрения позитивного замыкания в каждой из этих систем можно было бы оставить по одной ненулевой функции.

Завершая рассмотрение подмножеств множества (1), обратимся к одноэлементным системам $\{0\},\{(002)\},\{(010)\},\{(011)\},\{(022)\}$. Заметим, что множества

$$
\{0, x\}, \quad\{(002), x\}, \quad\{(011), x\}
$$

совпадают соответственно с множествами

$$
V_{01}^{(1)} \cap V_{12}^{(1)}, \quad T_{0}^{(1)} \cap T_{2}^{(1)} \cap V_{01}^{(1)}, \quad T_{0}^{(1)} \cap V_{10}^{(1)} .
$$

Поэтому, как и выше, множества (4) будут совпадать с множествами всех одноместных функций из позитивных замыканий систем (3). Это свойство является определяющим для позитивных замыканий систем $\{0\},\{(002)\},\{(011)\}$. О дальнейших расширениях данных систем функциями из (1) уже говорилось выше.

Остается отметить, что функции (010), (022) двойственны функциям (002), (011) относительно перестановки $2 x$.

Итак, из всех подмножеств множества (1) остается рассмотреть одноэлементные множества $\{(001)\}$ и $\{(020)\}$.

Перейдем к случаю, когда $Q$ есть подмножество одного из множеств $V_{01}^{(1)}, V_{02}^{(1)}, V_{10}^{(1)}$, $V_{12}^{(1)}, V_{20}^{(1)}, V_{21}^{(1)}$. Поскольку классы $V_{01}, \ldots, V_{21}$ попарно двойственны, мы рассмотрим, например, класс $V_{02}$.

Лемма 2. Каждая из систем функций $\{0,(101)\},\{(002),(101)\},\{(002), 1\},\{(101), 1\}$, $\{0,(010), 1\}$ позитивно порождает класс $V_{02}$.

Доказательство. Рассмотрим систему $\{0,(101)\}$. Функции (010), 1 получаются из функций $0,(101)$ суперпозициями. Далее,

$$
(y=(002)(x)) \equiv((010)(x)=x) \&(y=0) \vee((010)(x)=0) \&(y=x) .
$$


К системе $\{0,(101)\}$ сводится система $\{(002),(101)\}$, поскольку константа 0 получается в последней системе суперпозицией.

В случае системы $\{(002), 1\}$ сначала получаем константу 0 , а затем образуем функцию (101):

$$
(y=(101)(x)) \equiv((002)(x)=x) \&(y=1) \vee(x=1) \&(y=0) .
$$

В случае системы $\{(101), 1\}$ константа 0 получается очевидным образом. В случае системы $\{0,(010), 1\}$ образуем функцию (101):

$$
(y=(101)(x)) \equiv((010)(x)=0) \&(y=1) \vee(x=1) \&(y=0) .
$$

Лемма доказана.

Ввиду леммы 2, из подмножеств множества $V_{02}^{(1)}$, не содержащихся целиком в классе $T_{0}$, подлежат исследованию лишь следующие множества (мы опускаем функцию $x$, которая присутствует в любом позитивно замкнутом классе): $\{(101)\},\{1\},\{0,1\},\{(010), 1\}$. Однако множества $\{1\}$ и $\{(010), 1\}$ двойственны множествам $\{0\}$ и $\{0,(011)\}$ относительно перестановки $2 x+1$. Далее, справедливо соотношение

$$
\{0,1, x\}=V_{02}^{(1)} \cap V_{12}^{(1)} .
$$

Отсюда следует, что в позитивное замыкание системы $\{0,1\}$ из числа одноместных функций входят лишь функции $0,1, x$. Это свойство можно считать характеристическим для замыкания $\operatorname{Pos}[0,1]$. В силу леммы 2 , дальнейшие расширения системы $\{0,1\}$ функциями из $V_{02}^{(1)}$, отличными от $x$, приводят к множествам, позитивно полным в классе $V_{02}$.

Система $\{(101)\}$ будет рассмотрена в следующем разделе.

\section{4. Основной результат}

Применительно к целям нашего исследования рассмотрим частный случай общего понятия эндоморфизма универсальной алгебры [1]. Пусть $Q=\left\{g_{1}, \ldots, g_{s}\right\}-$ множество функций из $P_{3}, \varphi$ - отображение из $E_{3}$ в $E_{3}$ (не обязательно инъективное). Будем говорить, что $\varphi-$ эндоморфизм множества $Q$, если для любого $i, 1 \leqslant i \leqslant s$, и любых элементов $a_{1}, \ldots, a_{n_{i}}$ из $E_{3}$ выполняется соотношение

$$
g_{i}\left(\varphi\left(a_{1}\right), \ldots, \varphi\left(a_{n_{i}}\right)\right)=\varphi\left(g_{i}\left(a_{1}, \ldots, a_{n_{i}}\right)\right) .
$$

Из определения сразу следует, что эндоморфизм множества $Q$ является эндоморфизмом тождественной функции $x$ и любой суперпозиции функций из $Q$. Кроме того, композиция (суперпозиция) двух эндоморфизмов множества $Q$ также есть эндоморфизм множества $Q$. Легко понять, что тождественное отображение всегда является эндоморфизмом множества $Q$.

Обозначим через $\operatorname{End}(Q)$ совокупность всех эндоморфизмов множества $Q$. Из приведенные выше фактов следует, что $\operatorname{End}(Q)$ вместе с бинарной операцией композиции образует полугруппу отображений множества $E_{3}$ в себя с единицей, которой является тождественное отображение.

Лемма 3. Пусть $Q \subset P_{3}$ и функиия $g\left(x_{1}, \ldots, x_{n}\right)$ определяется позитивной формулой над $Q$. Тогда $\operatorname{End}(Q) \subseteq \operatorname{End}(g)$. 
Доказательство. Пусть позитивная формула $\Phi\left(x_{1}, \ldots, x_{n}, y\right)$ над множеством $Q$ определяет отношение $y=g\left(x_{1}, \ldots, x_{n}\right)$. Возьмем произвольный набор $\left(a_{1}, \ldots, a_{n}\right)$ из $E_{3}^{n}$ и пусть $b=g\left(a_{1}, \ldots, a_{n}\right)$. Тогда значение $\Phi\left(a_{1}, \ldots, a_{n}, b\right)$ истинно. Покажем, что для любого эндоморфизма $\varphi \in \operatorname{End}(Q)$ значение $\Phi\left(\varphi\left(a_{1}\right), \ldots, \varphi\left(a_{n}\right), \varphi(b)\right)$ также истинно. Отсюда будет следовать, что

$$
\varphi(b)=g\left(\varphi\left(a_{1}\right), \ldots, \varphi\left(a_{n}\right)\right)
$$

то есть $\varphi-$ эндоморфизм функции $g$.

Предположим, что $z_{1}, \ldots, z_{m}-$ все связанные переменные формулы $Ф$. Пусть

$$
t_{1}\left(x_{1}, \ldots, x_{n}, y, z_{1}, \ldots, z_{m}\right)=t_{2}\left(x_{1}, \ldots, x_{n}, y, z_{1}, \ldots, z_{m}\right)
$$

- равенство над $Q$, входящее в формулу $\Phi$, и $h_{1}, h_{2}-$ функции, реализуемые термами $t_{1}$, $t_{2}$. Будем далее предполагать, что при установлении истинности значения $\Phi\left(a_{1}, \ldots, a_{n}, b\right)$ связанные переменные $z_{1}, \ldots, z_{m}$ формулы $\Phi$ принимают значения $c_{1}, \ldots, c_{m}$, а равенство (5) выполняется на наборе $\left(a_{1}, \ldots, a_{n}, b, c_{1}, \ldots, c_{m}\right)$. Следовательно, в этом случае

$$
h_{1}\left(a_{1}, \ldots, a_{n}, b, c_{1}, \ldots, c_{m}\right)=h_{2}\left(a_{1}, \ldots, a_{n}, b, c_{1}, \ldots, c_{m}\right) .
$$

Поскольку функции $h_{1}, h_{2}$ реализуются суперпозициями функций из $Q$, либо совпадают с тождественной функцией и $\varphi \in \operatorname{End}(Q)$, при $i=1,2$ будут справедливы равенства

$$
h_{i}\left(\varphi\left(a_{1}\right), \ldots, \varphi\left(a_{n}\right), \varphi(b), \varphi\left(c_{1}\right), \ldots, \varphi\left(c_{m}\right)\right)=\varphi\left(h_{i}\left(a_{1}, \ldots, a_{n}, b, c_{1}, \ldots, c_{m}\right)\right) .
$$

Из (6), (7) выводим, что

$$
\begin{aligned}
h_{1}\left(\varphi\left(a_{1}\right), \ldots, \varphi\left(a_{n}\right), \varphi(b), \varphi\left(c_{1}\right)\right. & \left., \ldots, \varphi\left(c_{m}\right)\right) \\
& =h_{2}\left(\varphi\left(a_{1}\right), \ldots, \varphi\left(a_{n}\right), \varphi(b), \varphi\left(c_{1}\right), \ldots, \varphi\left(c_{m}\right)\right) .
\end{aligned}
$$

Таким образом, из выполнимости (5) на наборе $\left(a_{1}, \ldots, a_{n}, b, c_{1}, \ldots, c_{m}\right)$ вытекает выполнимость этого равенства на наборе $\left(\varphi\left(a_{1}\right), \ldots, \varphi\left(a_{n}\right), \varphi(b), \varphi\left(c_{1}\right), \ldots, \varphi\left(c_{m}\right)\right)$.

Это, в свою очередь, означает, что если при установлении истинности значения $\Phi\left(a_{1}, \ldots, a_{n}, b\right)$ в качестве значений связанных переменных $z_{1}, \ldots, z_{m}$ были выбраны значения $c_{1}, \ldots, c_{m}$, то для установления истинности $\Phi\left(\varphi\left(a_{1}\right), \ldots, \varphi\left(a_{n}\right), \varphi(b)\right)$ в качестве значений переменных $z_{1}, \ldots, z_{m}$ следует выбрать значения $\varphi\left(c_{1}\right), \ldots, \varphi\left(c_{m}\right)$. Лемма доказана.

Лемма 4. Пусть $g$ - одна из функиий (001), (101). Тогда множество всех одноместных функций из позитивного замыкания функиии $g$ состоит из функций $x, g(x), g(g(x))$.

Доказательство. Нетрудно убедиться в том, что $\operatorname{End}(\{(001)\})$ состоит только из функций $0,(001), x$. Для любой одноместной функции $h$, отличной от функций $0,(001), x$, находим такую функцию $\varphi$ из множества $\{0,(001)\}$, которая не является эндоморфизмом функции $h$. Если функция $h$ не сохраняет 0 , то в качестве функции $\varphi$ следует взять константу 0 . В противном случае в качестве функции $\varphi$ можно взять функцию (001). Далее применяем лемму 3.

Перейдем к функции (101). Проверяем, что $\operatorname{End}(\{(101)\})$ состоит только из функций (010), (101), x. Если $h(x) \in\{0,(002), 1\}$, то эндоморфизмом функции $h$ не является функция (101), во всех остальных случаях - функция (010). Лемма доказана.

Итогом проведенных выше исследований является следующая теорема. 
Теорема 1. $B P_{3}$ существует ровно 51 позитивно замкнутый класс, позитивно порождаемый множеством одноместных функиий. Это 26 классов, позитивно порождаемых единственной одноместной функиией (включая позитивно предполный класс $S_{x+1}$ и класcbl $\left.S_{2 x}, S_{2 x+2}, S_{2 x+1}\right)$, и 25 классов, позитивно порождаемых двумя одноместными функцияли: класс $P_{3}$, позитивно предполные классы $T_{0}, T_{1}, T_{2}, V_{01}, V_{02}, V_{10}, V_{12}, V_{20}, V_{21}$, классы $T_{01}, T_{02}, T_{12}$, а также 12 классов, позитивно порождаемых системами функиий $\{0,(002)\},\{0,(011)\},\{0,(022)\},\{0,1\},\{0,2\},\{(002), 2\},\{(010), 1\},\{(011), 1\},\{1,(212)\}$, $\{1,2\},\{(112), 2\},\{(212), 2\}$.

\section{Список литературы}

1. Кон П., Универсальная алгебра. Мир, Москва, 1968.

2. Марченков С. С., О выразимости функций многозначной логики в некоторых логикофункциональных языках. Дискретная математика (1999) 11, №4, 110-126.

3. Марченков С. С., Критерий позитивной полноты в трехзначной логике. Дискретный анализ $u$ исследование операщий. Сер. 1 (2006) 13, №3, 27-39.

4. Марченков С. С., Дискриминаторные позитивно замкнутые классы трехзначной логики. Дискретный анализ и исследование операщий. Сер. 1 (2007) 14, №3, 53-66.

5. Яблонский С. В., Введение в дискретную математику. Наука, Москва, 1986.

6. Янов Ю. И., Мучник А. А., О существовании $k$-значных замкнутых классов, не имеющих базиса. Доклады АН СССР (1959) 127, №1, 44-46.

7. Post E. L., Introduction to a general theory of elementary propositions. Amer. J. Math. (1921) 43, $163-185$.

8. Post E. L., Two-valued iterative systems of mathematical logic. Princeton Univ. Press, Princeton, 1941.

Статья поступила 9.10.2008. 